\title{
Estimación de las relaciones entre la inversión en medios digitales y las variables financieras de la empresa: una aproximación para Colombia
}

FECHA DE RECEPCIÓN: 17 de enero FECHA DE APROBACIÓN: 1 de febrero Pp. $12-25$

\section{Resumen}

Este artículo realiza una primera estimación de la relación entre la inversión en medios digitales y algunas variables financieras sectoriales en Colombia. En primera medida, se hace una revisión de la literatura relacionada con el impacto del marketing y del marketing digital en el desempeño de las empresas. A continuación, se realiza un análisis de variables sectoriales de liquidez, rentabilidad, endeudamiento y concentración en los sectores de alimentos, aseo personal, automotriz, bebidas y tabaco, construcción, diversión, muebles, servicios, telecomunicaciones, turismo y vestuario utilizando la técnica de mínimos cuadrados ordinarios (MCO) para los años 2011, 2012, 2013 y 2014. Para el análisis, también se toma la inversión en medios digitales para esos mismos periodos.

\section{Palabras clave}

E- marketing, indicadores financieros, inversión en medios digitales, Mínimos Cuadrados Ordinarios, nivel de endeudamiento.

\footnotetext{
Amalia Novoa Hoyos

Economista, magíster en Finanzas, docente asociada de la Universidad EAN.
}

Mauricio Sabogal Salamanca

Economista, magíster en Mercadeo, docente asociado de la Universidad EAN.

\section{Camilo Vargas Walteros}

Economista, magíster en Economía, docente asociado de la Universidad EAN. 


\title{
Relations Estimate between Investment in Digital Media and financial Variables of Companies: a Colombian Overview
}

\begin{abstract}
Abstrac. This article shows a first estimate about the relationship between investment in digital media and some financial variables in Colombia. First, a literature review is made about the impact of marketing and digital marketing in Company performance. Then, an analysis of the sectorial variables such as liquidity, profitability, indebtedness and concentration in sectors like food, personal grooming, automotive, drinking and tobacco, construction, entertainment, furniture, services, telecommunication, tourism and clothing using the technique of ordinary squared minimums (OSM) in the years 2011, 2012, 2013 and 2014. For this study, investment in digital media in the above- mentioned years is also taken into account.
\end{abstract}

Key words. E- marketing, financial indicators, investment in digital media, ordinary squared minimums, indebtedness level.

\section{Estimation des relations entre l'investissement en moyens technologiques et les variables financières des entreprises: une approximation pour la Colombie}

Resumé. Cet article réalise une première estimation, pour la Colombie, de la relation entre l'investissement en outils technologiques et digitaux et certaines variables financières sectorielles. Nous commencerons par réaliser une rétrospective de la littérature relative à l'impact du marketing et du marketing digital sur la performance des entreprises pour ensuite réaliser une analyse des variables sectorielles prenant en compte la liquidité, la rentabilité, l'endettement et la concentration dans les secteurs de l'alimentation, de la cosmétique, de l'automobile, des boissons et du tabac, de la construction, du divertissement, de l'ameublement, des services, des télécommunications, du tourisme en utilisant la technique de minimums carrés ordinaires (MCO) pour les années 2011, 2012, 2013 et 2014. L'investissement en outils technologiques et digitaux a également été pris en compte lors de l'analyse pour les mêmes périodes.

Mots clefs. E-marketing, indicateurs financiers, investissement en outils technologiques et digitaux, minimums des carrés ordinaires (MCO), niveau d'endettement.

\section{A estimativa das relações entre o investimento em mídia digital e as variáveis financeiras da empresa: uma abordagem para Colômbia}

Resumo. Este artigo faz uma primeira estimativa da relação entre o investimento em mídias digitais e algumas variáveis financeiras setoriais na Colômbia. Em uma primeira etapa, uma revisão da literatura sobre o impacto do marketing e marketing digital desempenho das empresas é feita. Em seguida, se realiza uma análise de variáveis setoriais de liquidez, rentabilidade, endividamento e concentração nos setores de alimentos, cuidados pessoais, automóveis, bebidas e tabaco, construção, entretenimento, móveis, serviços, telecomunicações, turismo e vestuário usando a técnica de mínimos quadrados ordinários (MQO) para os anos de 2011, 2012, 2013 e 2014. Para essa análise também se considera o investimento em mídia digital para os mesmos períodos.

Palabras chave. E- marketing, indicadores financeiros, investimento em mídia digitais, mínimos quadrados ordinários, nível de endividamento. 


\section{Introducción}

Tl consumidor ha cambiado gracias a Cla aparición de las nuevas tecnologías digitales. Ahora es más exigente, ya que posee más información sobre los productos disponibles en Internet, y se relaciona constantemente con otros usuarios en la web para intercambiar opiniones sobre productos y servicios. El proceso de compra también ha cambiado, ya que no todo sucede en la tienda. El cliente busca previamente información en la web, muchas veces antes de comprar, y puede utilizar las plataformas online más de una vez antes y después de tomar su decisión de compra (Strauss, y Frost, 2012).

Lo anterior ha hecho que en la sociedad actual sea cada vez más importante el marketing digital. Hay una tendencia creciente a su utilización por parte de las empresas, derivado de los cambios de este consumidor, lo que ha significado que aumente su utilización en los últimos años. De hecho, para Shellen Shum, analista de eMarketer, las inversiones tradicionales en TV, periódicos y revistas se han visto afectadas negativamente por las crecientes inversiones en formatos digitales. El reporte de eMarketer también menciona un crecimiento sostenido del mercado publicitario online, que para el 2015 crecerá un 18\% en todo el mundo (eMarketer, 2015).

El marketing digital tiene ciertas ventajas sobre los medios tradicionales, entre otras, permite medir los resultados de manera más precisa que el marketing tradicional, así mismo, permite desarrollar acciones con impacto más global, acciones de mercadeo 24/7 y la utilización de recursos de manera más focalizada y segmentada, a un costo menor, en relación con los medios tradicionales (Strauss, y Frost, 2012). A pesar de estas ventajas del marketing digital, sobre el tradicional, en estos momentos la literatura cuenta con pocos estudios que aborden la relación entre la inversión en medios digitales y el desempeño financiero de la compañía, que es uno de los principales motores para generar valor a los accionistas a través de la maximización del valor de la compañía (García, 2009).

El presente artículo será un aporte a la investigación a cerca de la relación entre la inversión en medios digitales y el desempeño empresarial medido a través de algunas variables financieras. Para conseguir el objetivo, se utilizaron indicadores financieros agregados de diferentes sectores de la economía colombiana, para varios años, junto con los datos de inversión de medios digitales. Se realizaron varias regresiones con el fin de establecer algunas relaciones entre las variables en mención. El artículo se encuentra dividido en cinco partes: la primera, es la introducción; la segunda, se refiere a un panorama de la literatura en términos de la relación entre marketing, marketing digital y el desempeño financiero de la compañía; la tercera parte, muestra el diseño metodológico del artículo; la cuarta presenta los resultados, y la quinta muestra las conclusiones. 


\section{Relación entre marketing, marketing digital y desempeño de la empresa}

\subsection{Definición de marketing y E-Marketing}

Una primera definición de marketing es la dada por la Asociación Americana del Marketing en Kotler y Keller (2012), en donde el marketing es «la actividad o grupo de entidades y procedimientos para crear, comunicar, entregar e intercambiar ofertas que tienen valor para los consumidores, clientes, socios y la sociedad en general». Una definición más orientada hacia esta disciplina con un componente social también es mencionada en Kotler y Keller (2012), donde se concibe al marketing como «un proceso social por el cual tanto grupos como individuos obtienen lo que necesitan y desean mediante la creación, oferta y libre intercambio de productos y servicios con otros grupos de individuos». Por su parte, Chaffey y Chadwick definen el marketing digital como la ejecución del marketing utilizando medios electrónicos como la web, e-mail, televisión interactiva y medios inalámbricos junto con datos digitales de las características y comportamiento de los clientes (Chaffey \& Ellis-Chadwick, 2014).

Esta nueva definición de marketing no solamente tiene que ver con el cambio de plataforma para la comunicación y la entrega de valor a los clientes. Con más de tres mil millones de personas que tienen p.159 acceso a Internet, la sociedad
vive un tiempo de sobrecarga de información que ha cambiado el panorama del marketing. Un gran cambio ha sido la transición de un modelo de comunicación masiva en donde los encargados de marketing le decían a los consumidores que era lo mejor para ellos, a una conversación más personal, móvil, social y por supuesto, online, entre marcas y consumidores. Esto también implica que la discusión ya no sea sobre si los presupuestos incluyen rubros digitales, sino cuál será la cantidad apropiada de inversión en medios digitales, y cómo se integrarán apropiadamente los mundos offline o tradicional, y el online, al igual que cómo crear una participación y un «ruido» -buzzrelevante en online (Budukova, 2014).

\subsection{Relación entre el marketing y el desempeño de la compañía}

Autores como Pimenta da Gamma (2011) han mostrado la dificultad que representa el medir el desempeño del marketing, entre otras razones, porque tradicionalmente, este, en su práctica, ha enfatizado en su lado creativo, a expensas de su lado analítico, lo que en muchas organizaciones es un reflejo de la incapacidad de justificar los recursos empleados y cuantificar sus correspondientes efectos.

A pesar de lo anterior, la literatura ha desarrollado diferentes aproximaciones para la medición del desempeño del marketing y su relación con el desempeño de la empresa. Frösen et al (2013) realiza un recuento histórico de las diferentes aproximaciones a la medición del desempeño del marketing; dicha medición en sus inicios estuvo relacionada con un concepto de auditoría en los años 60, acompañada también por un enfoque 
basado en la eficiencia del marketing y, más adelante en esa misma década, en las métricas financieras como las ganancias y el flujo de caja, como variables clave para medir el desempeño de las actividades de marketing. En los años 70 y 80, de acuerdo con este mismo autor, la medición basada únicamente en el desempeño financiero se movió hacia una visión multidimensional, utilizando variables internas y externas adicionales a las financieras, y, más recientemente, este enfoque fue orientado hacia variables no financieras como la satisfacción del cliente, la lealtad y el Brand Equity, como métricas para entender el desempeño.

La visión más moderna de las métricas del desempeño del marketing buscan entender su actividad como la combinación de diferentes tipos de variables, financieras y no financieras (Frösen et al, 2013, 718). Pimenta da Gamma (2011) propone una medición basada en un modelo de desempeño que tenga en cuenta variables de diverso tipo, que interactúan con los procesos y la cultura de la compañía, para generar un resultado financiero medido en términos de ventas, ganancias, márgenes y flujos de caja.

No obstante esta nueva multidimensionalidad en el análisis, las finanzas en la evaluación del desempeño del marketing siguen siendo relevantes. Cravens (2006), menciona que la productividad del marketing y su medición debe ser un área de alta prioridad según el Marketing Science Institute.

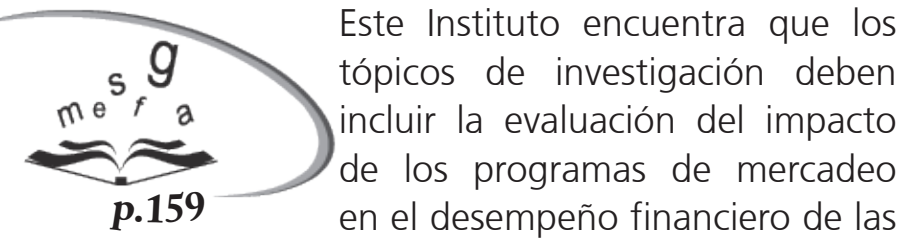
organizaciones, la utilización del ROI para el mejor uso de los recursos, valorar los intangibles como el Brand Equity o el Customer Equity, enlazar resultados o variables intermedias de la actividad de marketing, tales como el awareness o la lealtad de los clientes, con resultados financieros, determinar el efecto de largo plazo de los programas de mercadeo y evaluar el efecto de la publicidad en las ventas, el Premium de precios cobrado por las compañías y la efectividad en las ventas.

Con relación al marketing digital, la literatura cuenta con pocos artículos que se aproximen a esta relación. En la actualidad existe una discusión sobre cómo conseguir una mayor efectividad del marketing digital por medio del uso del Big Data, los servicios en la nube y la utilización apropiada de los datos de comportamiento del cliente (behavioralbased data). Estas herramientas tecnológicas permiten, a través de métodos cuantitativos, entender mejor cómo toman decisiones los clientes con el fin de segmentarlos mejor $y$, de este modo, invertir de manera más precisa e inteligente los presupuestos de marketing, a la par con la creación de comunicaciones y experiencias personalizadas. La calidad de los datos de las compañías y el apalancamiento en su uso en todos los canales, su almacenamiento y uso instantáneo en la nube, así como la integración entre actividades online y offline y la excelencia de su ejecución, son los retos futuros que enfrentarán las compañías en la esfera digital (Budikova, 2014).

Así mismo, existe una discusión alrededor del marketing interactivo; por ejemplo, a través de todos los tipos de canales que el cliente utilice o desee utilizar, que es justamente la definición de omnichannel; o la omnipresencia de la marca en todos los canales para el cliente. En este contexto, las marcas deben garantizar que haya una serie de acciones de marketing que involucran al consumidor de manera activa, en la tienda, en el smartphone, en las redes sociales, en 
el sitio de e-Commerce, en buscadores, y en cualquier otro lugar donde el consumidor esté. De igual forma, este concepto de always on ya no se refiere al concepto de campaña de marketing convencional, sino, por el contrario, desarrolla las habilidades necesarias para que el cliente interactúe con la compañía de manera dinámica, a través de todos los canales. Esto implica el manejo de grandes volúmenes de información en tiempo real, algo que solo puede lograrse con una robusta inteligencia de negocios, entendida esta como la combinación de tecnologías, arquitecturas, gente, procesos y metodologías que transformen datos en información útil (Stone and Woodcock, 2014).

En la revisión de la literatura solo se encontró un estudio que busca medir de alguna manera el impacto del marketing digital en el desempeño empresarial. Uribe, Rialp, \& Llonch, J. (2013), elaboran una evaluación, utilizando ecuaciones estructurales, en la cual buscan entender las redes sociales como una herramienta que afecta el desempeño de las empresas españolas. Los resultados indican que la utilización intensiva y el esfuerzo dedicado en actividades de mercadeo de redes sociales, influyen en el desempeño de la empresa a través de un constructo llamado orientación al mercado. De acuerdo con estos autores, una verdadera e intensa estrategia de marketing de redes sociales es necesaria para impactar realmente la operación de la empresa y obtener mejores resultados.

A pesar de lo anterior no se encontraron estudios que midan la relación entre la inversión en medios digitales y el desempeño de las organizaciones, y más aún cuando se emplean datos sectoriales para tratar de entender esta relación, algo que se realiza en este artículo.

\section{Diseño metodológico}

E I análisis desarrollado en esta investigación es de carácter cuantitativo, con un enfoque exploratorio y descriptivo, donde se pretende entender la relación que existe entre la inversión en medios digitales y algunas variables financieras de las empresas, utilizando para esto una muestra específica de sectores, a través de la técnica de regresión con mínimos cuadrados ordinarios (MCO).

En cuanto a la literatura relevante, actualizada y aplicable al caso colombiano, Islamoglu y Celik (2015) analizaron los determinantes de la rentabilidad de las firmas que cotizan en la bolsa de Turquía. Al igual que muchos documentos en ese artículo la rentabilidad empresarial es calculada utilizando el retorno sobre ventas -margen bruto-, retorno sobre activos (ROA) y retorno sobre el patrimonio (ROE). Estos autores también realizan una revisión extensiva de los determinantes de la rentabilidad empresarial y en su artículo emplean indicadores financieros de liquidez -prueba ácida-, estructura de capital -capital como proporción de los activos-, participación de mercado -ventas como proporción de las ventas totales- entre otras.

Allí se utiliza un análisis de regresión de datos panel, pero en este estudio se aplica la metodología de Mínimos Cuadrados Ordi-narios (MCO) debido a su sencillez en 
la aplicación, la estructura de los datos -no son datos panel dado que no son empresas sino sectores económicos- y en ciertas situaciones MCO produce resultados similares en comparación a métodos más sofisticados.

\subsection{Descripción de datos y variables}

Como fuentes del modelo de regresión se eligió una muestra de nueve sectores en Colombia, así (Tabla 1):

Tabla 1. Sectores económicos en Colombia

\begin{tabular}{|l|l|}
\hline \multicolumn{1}{|c|}{ Columna 1 } & \multicolumn{1}{c|}{ Columna 2 } \\
\hline Alimentos & Muebles \\
\hline Aseo personal & Servicios \\
\hline Automotriz & Telecomunicaciones \\
\hline Bebidas y tabaco & Turismo \\
\hline Construcción & Vestuario \\
\hline Diversión & \\
\hline
\end{tabular}

Fuente. Elaboración propia de los autores, a partir de información de EMIS e IAB Colombia.

Los datos contienen los montos de inversión acumulada en medios digitales por parte de estos sectores referenciados en el informe sobre la inversión en medios digitales en Colombia (IAB Colombia, varios años). Los datos sectoriales que evidencian el desempeño financiero de estos sectores se obtuvieron de la base de datos Emis (EMIS, 2015).

La inversión acumulada en medios digitales (IMD), fue calculada como el porcentaje de participación de la inversión del sector dentro del total de inversión en medios digitales. Esta inversión fue estimada en $\$ 111.942$ millones en 2014 de los cuales el 60,48\% es representado por los sectores de alimentos, aseo personal, automotriz, bebidas y tabaco, construcción, diversión, muebles, servicios, telecomunicaciones, turismo y vestuario (IAB Colombia, varios años).

Posteriormente se agruparon las siguientes variables de acuerdo con su clasificación de deuda, liquidez, rentabilidad y concentración (Tabla2; Tabla 3; Tabla 4; Tabla 5):

\section{Tabla 2. Indicadores de deuda}

\begin{tabular}{|l|l|}
\hline \multicolumn{1}{|c|}{ Indicador } & \multicolumn{1}{|c|}{ Descripción } \\
\hline $\begin{array}{l}\text { Apalancamiento } \\
\text { (APA) }\end{array}$ & $\begin{array}{l}\text { Comprende la relación de } \\
\text { endeudamiento, en contraste } \\
\text { con sus niveles de patrimonio. }\end{array}$ \\
\hline $\begin{array}{l}\text { Endeudamiento } \\
\text { (END) }\end{array}$ & $\begin{array}{l}\text { Comprende la relación de } \\
\text { endeudamiento de los sec- } \\
\text { tores versus su nivel de activos. }\end{array}$ \\
\hline $\begin{array}{l}\text { Relación pasivo/ } \\
\text { Ventas (PAVE) }\end{array}$ & $\begin{array}{l}\text { Comprende la relación entre } \\
\text { el pasivo y las ventas de los } \\
\text { sectores. }\end{array}$ \\
\hline
\end{tabular}

Fuente. Elaboración propia de los autores.

Tabla 3. Indicadores de liquidez

\begin{tabular}{|l|l|}
\hline \multicolumn{1}{|c|}{ Indicador } & \multicolumn{1}{c|}{ Descripción } \\
\hline Prueba ácida & Comprendida como la capacidad \\
(Quick Ratio & de atender los compromisos de \\
-QR-) & deuda de corto plazo con activos \\
& de fácil convertibilidad a efectivo. \\
\hline
\end{tabular}

Fuente. Elaboración propia de los autores. 
Tabla 4. Indicadores de rentabilidad

\begin{tabular}{|l|l|}
\hline \multicolumn{1}{|c|}{ Indicador } & \multicolumn{1}{c|}{ Descripción } \\
\hline Margen Neto (MN) & $\begin{array}{l}\text { Comprende la relación entre la utilidad neta promedio de los } \\
\text { sectores y su nivel de ventas. }\end{array}$ \\
\hline Margen Operativo (MO) & $\begin{array}{l}\text { Comprende la relación entre la utilidad operacional promedio de } \\
\text { los sectores y su nivel de ventas. }\end{array}$ \\
\hline ROA & $\begin{array}{l}\text { Comprende la relación entre los resultados financieros de los } \\
\text { sectores y el nivel de activos. }\end{array}$ \\
\hline ROE & $\begin{array}{l}\text { Comprende la relación entre los resultados financieros netos del } \\
\text { sector -utilidad neta- y su nivel de patrimonio. }\end{array}$ \\
\hline
\end{tabular}

Fuente. Elaboración propia de los autores.

Tabla 5. Indicadores de concentración

\begin{tabular}{|c|l|}
\hline \multicolumn{1}{|c|}{ Indicador } & \multicolumn{1}{|c|}{ Descripción } \\
\hline Índice de Herfindahl Hirschman $(\mathrm{IHH})$ & $\begin{array}{l}\text { Se calculó a partir del reporte de EMIS Benchmark Key } \\
\text { Indicators (EMIS, 2015), como la participación de cada } \\
\text { empresa dentro de las ventas más representativas del sector } \\
\text { posteriormente se eleva este porcentaje al cuadrado y se } \\
\text { realiza la suma. }\end{array}$ \\
\hline
\end{tabular}

Fuente. Elaboración propia de los autores.

La muestra total comprende cuatro años de información (2011, 2012, 2013 y 2014) y 11 sectores para un total de 44 observaciones para cada una de las nueve variables descritas anteriormente.

\subsection{Ecuaciones del modelo}

Inicialmente se estimó el impacto de la inversión en medios digitales sobre el desempeño empresarial. Este objetivo se desarrolló mediante una regresión lineal simple entre los indicadores de rentabilidad como variables dependiente y la inversión en medios digitales como variable independiente las cuales se describen de acuerdo a la siguiente ecuación:

\section{Regresiones de rentabilidad}

$$
R_{i}={ }_{0}+{ }_{1} I M D+u_{1 i}
$$

$R$ representa el indicador de rentabilidad -margen operativo, margen neto, ROA y ROEe IMD muestra el porcentaje de inversión en medios digitales.

Posteriormente se estimaron los determinantes financieros de la inversión en medios digitales utilizando una regresión lineal simple. En este caso la variable dependiente es la inversión en medios digitales y las variables independientes incorporan las variables agrupadas en los indicadores de deuda, liquidez y concentración:

\section{Regresiones de deuda}

$$
I M D_{i}={ }_{0}+{ }_{1} E+u_{2 i}
$$

IMD representa el porcentaje de inversión en medios digitales y $E$ un indicador de deuda -endeudamiento, apalancamiento, pasivo y relación pasivo ventas-. 


\section{Regresión de liquidez}

$$
I M D_{i}={ }_{0}+{ }_{1} L+u_{3 i}
$$

En la ecuación (3) -IMD- representa el porcentaje de inversión en medios digitales y $L$ un indicador de liquidez -prueba ácida-.

\section{Regresión de concentración}

$$
I M D_{i}={ }_{0}+{ }_{1} C+u_{4 i}
$$

En la ecuación (4) IMD representa el porcentaje de inversión en medios digitales y $C$ un indicador de concentración -índice de Herfindahl Hirschman-.

\section{Análisis de resultado}

L os resultados de las regresiones en las cuales la inversión en medios digitales es la variable independiente y el indicador de rentabilidad es la variable dependiente, se encuentran en las ecuaciones 5 a 8 . Es importante aclarar que dada la alta multicolinealidad entre los diferentes indicadores del grupo de rentabilidad, se realizaron regresiones simples -en las ecuaciones 5 a 13 los errores estándar se presentan entre paréntesis y el programa utilizado es Eviews versión 7.0-:

\section{Regresiones de rentabilidad}

$$
\begin{aligned}
& M N_{i}=\underset{(0,0132)}{0,0433}+\underset{(0,1988)}{0,2865 I M D} \\
& M O_{i}=\underset{(0,0194)}{0,0910-\underset{(0,2914)}{0,0432} I M D} \\
& R O A_{i}=0,0435+0,0628 I M D \\
& (0,0075) \quad(0,1131) \\
& R O E_{i}=\underset{(0,0111)}{0,0739}+\underset{(0,1670)}{0,3087} I M D
\end{aligned}
$$

Los $p$ valores y el $R$ cuadrado ajustado de las regresiones de rentabilidad se encuentran más adelante (Tabla 6). Los resultados indican que con un nivel de significancia del 5\%, ninguna regresión es significativa.
Tabla 6. Indicadores estadísticos de las regresiones de rentabilidad

\begin{tabular}{|c|c|c|}
\hline Regresión & Valor $\mathbf{P}$ & $\begin{array}{c}\text { R-Cuadrado } \\
\text { ajustado }\end{array}$ \\
\hline 5 & $15,69 \%$ & $2,44 \%$ \\
\hline 6 & $88,27 \%$ & $-2,32 \%$ \\
\hline 7 & $58,15 \%$ & $-1,63 \%$ \\
\hline 8 & $7,16 \%$ & $5,32 \%$ \\
\hline
\end{tabular}

Fuente. Elaboración propia de los autores.

La regresión (8) es la más robusta en términos estadísticos. Cuando la inversión en medios digitales es la variable independiente la rentabilidad medida a través del ROE es explicada en un 5,3\% y su impacto sobre el desempeño empresarial es del 0,3087\%. Cabe aclarar que esta regresión es significativa al $10 \%$.

Los resultados de las regresiones en las cuales la inversión en medios digitales es la variable dependiente y los indicadores de deuda son las variables independientes se muestran en las ecuaciones (9) a (11). 


\section{Regresiones de deuda}

$$
\begin{aligned}
& I M D_{i}=\underset{(0,0270)}{-\underset{(0,0542)}{0,0241}} \underset{\text {, }}{0,1637} E N D \\
& I M D_{i}=\underset{(0,0136)}{0,0105}+\underset{(0,0123)}{0,0440} A P A \\
& I M D_{i}=\underset{(0,0092)}{0,0236}+\underset{(0,0130)}{0,0536} P A V E
\end{aligned}
$$

Los $p$ valores y el $R$ cuadrado ajustado de las regresiones de deuda se presentan a continuación (Tabla 7). Los resultados indican que con un nivel de significancia del $1 \%$, todas las regresiones son significativas.

Tabla 7. Indicadores estadísticos de las regresiones de deuda

\begin{tabular}{|c|c|c|}
\hline Regresión & Valor P & $\begin{array}{c}\text { R-Cuadrado } \\
\text { ajustado }\end{array}$ \\
\hline 9 & $0,43 \%$ & $15,9 \%$ \\
\hline 10 & $0,09 \%$ & $21,5 \%$ \\
\hline 11 & $0,02 \%$ & $26,8 \%$ \\
\hline
\end{tabular}

Fuente. Elaboración propia de los autores.

La regresión (11) es la más robusta en términos estadísticos. Cuando la relación pasivo ventas, es la variable independiente su impacto sobre la inversión en medios digitales es del $0,0536 \%$ y tiene un poder explicativo del $26,8 \%$.

Los resultados de las regresiones en las cuales la inversión en medios digitales es la variable dependiente y el indicador de liquidez y concentración son las variables independientes son los siguientes:

Regresiones de liquidez

$$
I M D_{i}=\underset{(0,0275)}{0,0028} \underset{(0,02853)}{0,0563} Q R
$$

\section{Regresiones de concentración}

$$
I M D_{i}=\underset{(0,0085)}{0,0,0801-0,2076)} \underset{(0,065)}{0}
$$

Los $p$ valores y el $R$ cuadrado ajustado de las regresiones en las cuales la inversión en medios digitales es la variable dependiente, y el indicador de liquidez y concentración son las variables independientes, se encuentran más (Tabla 8). La regresión (12) es significativa al $10 \%$, mientras que la regresión (13), es significativa al $1 \%$ :

Tabla 8. Indicadores estadísticos de la regresión de liquidez y la regresión de concentración

\begin{tabular}{|c|c|c|}
\hline Regresión & Valor $\mathbf{P}$ & $\begin{array}{c}\text { R-Cuadrado } \\
\text { ajustado }\end{array}$ \\
\hline 12 & $5,47 \%$ & $0,14 \%$ \\
\hline 13 & $6,3 \%$ & $20 \%$ \\
\hline
\end{tabular}

Fuente. Elaboración propia de los autores.

La regresión (13) es la más robusta en términos estadísticos. Cuando el índice de Herfindahl Hirschman es la variable independiente su impacto sobre la inversión en medios digitales es del $-0,2076 \%$ y tiene un poder explicativo del $20 \%$.

En conclusión, al considerar el $\mathrm{p}$ valor y el $R$ cuadrado ajustado de las regresiones anteriores, las regresiones de deuda son las más robustas. Una posible interpretación de este resultado es que cuando las empresas enfrentan un mayor endeudamiento, tienen unos mayores incentivos a producir ventas, lo cual las hace incrementar su proporción de inversión en marketing, y, por ende, en medios digitales.

La inversión en medios digitales también guarda una relación con el índice de concentración de Herfindahl Hirschman o $\mathrm{IHH}$. 
A menor concentración del sector se presenta una mayor proporción de inversión en medios digitales. Esto puede estar explicado por dos razones: la primera, a mayor competencia hay una tendencia a invertir más en medios digitales; y la segunda, las empresas que se encuentran en sectores con bajo nivel de concentración, tienden a ser más pequeñas; y la proporción de inversión en medios digitales con relación a la inversión total en marketing podrá ser más alta que en empresas con niveles más altos de concentración y tamaños relativos más grandes.

Si unimos los resultados anteriores, se podría argumentar que dado que la competencia es más fuerte -regresión 13-, la diferenciación de producto adquiere mayor importancia y las compañías adquieren un mayor endeudamiento con el fin de incrementar su publicidad en medios digitales -regresiones 9,10 y 11 -

\subsection{Verificación de supuestos}

Se realizaron las pruebas de Breusch-PaganGodfrey (BPG), Harvey (H), Glejser (G) y White
(W) utilizando los estadísticos F y Chi-cuadrado para detectar heteroscedasticidad en las regresiones, de deuda. En estas regresiones y para todas las pruebas, se aceptó la hipótesis nula de errores homoscedásticos con un nivel de significancia del $1 \%-p$ valor más bajo de $15,81 \%-$.

Mediante el estadístico de Jarque-Bera, se aceptó la hipótesis nula que los errores siguen la distribución normal utilizando un nivel de significancia del $1 \%$ en todos los modelos $-p$ valor más bajo de 51,95\%-.

La hipótesis nula de correcta especificación del modelo fue aceptada con un nivel de significancia del $1 \%$ en todos los modelos, al aplicar la prueba de Ramsey - $p$ valor más bajo de $11,91 \%$ sin incluir la regresión (9) en la cual el $p$ valor es del 1,28\%-.

Al aplicar la prueba de causalidad de Granger, se acepta la hipótesis nula por tanto la causalidad entre «la relación pasivo ventas» y la inversión en medios digitales es de doble sentido -p valor más bajo de 6,28\%-. 


\section{Conclusiones}

E I marketing ha evolucionado a la par con la tecnología y el nuevo comportamiento de los clientes. Estos toman decisiones en contextos en donde interactúan con la tienda y con la web, lo que ha hecho que las empresas presten más atención a los medios digitales y los incluyan cada vez más dentro de su portafolio de inversión publicitaria. Lo anterior ha significado un crecimiento en los medios digitales a lo largo de los últimos años.

La relación entre el marketing y el desempeño de la compañía siempre ha sido una prioridad. Ha experimentado una evolución, desde el concepto de auditoría de los años 60, hasta la concepción del resultado financiero de una compañía como un modelo multidimensional, donde las decisiones de marketing producen unos resultados en variables intermedias como el awareness del consumidor, que al final se ven traducidas en resultados financieros. No obstante, las finanzas siguen siendo un punto clave para entender el logro de los objetivos de la compañía, luego, sigue siendo válido enlazar el marketing digital, con el desempeño financiero de una empresa.

Dentro de la literatura sobre marketing digital y desempeño de las organizaciones, hay una discusión sobre la asertividad del marketing, si se utilizan apropiadamente las tecnologías de segmentación por comportamiento y se logra ofrecer una experiencia individual y Omnichannel p.159 a cada cliente, de manera
Existen pocos estudios que examinan la relación entre marketing digital y desempeño de la empresa, siendo esta una oportunidad para medir mediante un enfoque econométrico, la relación que existe entre la inversión en medios digitales y los principales indicadores financieros que miden el desempeño de las empresas.

La primera conclusión del análisis es la relación positiva encontrada entre los indicadores de rentabilidad y la inversión en medios digitales. El que tiene mayor poder explicativo sobre la inversión en medios digitales es la Rentabilidad sobre el Equity (ROE), esta relación es estadísticamente significativa al $8 \%$. Ante un incremento, $1 \%$ en la inversión en medios digitales, en promedio el ROE aumenta en 0,3087\% -el poder explicativo de la inversión en medios digitales es del 5,3\%-.

Los indicadores de deuda -endeudamiento, apalancamiento y relación pasivo/ventasmuestran una relación positiva frente a la inversión en medios digitales, con un poder explicativo que oscila entre $15,9 \%$ y $26,8 \%$ dependiendo del indicador. Ante un incremento de $1 \%$ en la relación pasivo/ventas en promedio la inversión en medios digitales aumenta en $0,0536 \%$. Esta relación es estadísticamente significativa al 1\%, siendo este el indicador que presenta una relación más fuerte con la inversión en medios digitales.

La inversión en medios digitales y la concentración del sector están relacionadas negativamente. Ante un incremento del 1\% en el índice de Herfindahl Hirschman $(\mathrm{IHH})$ en promedio la inversión en medios digitales disminuye en 0,2076\% (el poder del IHH es del 20\%). Esta relación es estadísticamente significativa al $1 \%$. 
De acuerdo con los resultados anteriores, un alto nivel de endeudamiento probablemente genera más presión sobre los directivos y accionistas para la creación de recursos vía ventas, con el propósito de mejorar su situación financiera. Lo anterior, probablemente lleva a las empresas a incrementar sus niveles de inversión en mercadeo, en este caso, en medios digitales.

Con relación a los resultados de las regresiones se podría argumentar que la deuda es un determinante financiero de la inversión en medios digitales, porque cuando las empresas enfrentan una menor concentración $(\mathrm{IHH})$, la competencia es más fuerte, la diferenciación de producto adquiere mayor importancia y las compañías adquieren un mayor endeudamiento con el fin de incrementar su publicidad en medios digitales. Este resultado también se puede presentar para sectores con bajo índice de concentración, dado que las empresas que los componen tienen tamaños relativos menores y su proporción de inversión en medios digitales, será mayor en comparación con las empresas más grandes ubicadas en sectores con altos índices de concentración.

\section{Oportunidades y limitaciones}

E ste artículo realizó un aporte a la literatura $\checkmark$ de los determinantes del desempeño empresarial dado que en comparación a otros trabajos se incorporó la inversión en medios digitales. Esta fortaleza también es su mayor limitación, dado que en Colombia la información disponible para esta variable es muy limitada y como consecuencia, restringe las conclusiones del análisis de regresión, pero también la aplicación de métodos o variables alternativas.

Con el paso del tiempo se puede obtener más información de la inversión en medios digitales y de esta manera se puede enriquecer el análisis con variables macroeconómicas -por ejemplo PIB-. De otro lado, se pueden realizar encuestas a nivel microeconómico que investiguen sobre las variables específicas que determinan el desempeño empresarial.

Se ha dicho que las mediciones de las métricas del marketing que solo tienen en cuenta variables financieras como ventas, ganancias y márgenes; son estáticas y sólo miran el desempeño hacia el pasado, ignorando el valor de la firma en el largo plazo (Frösen et al, 2013, 717). Por tal razón, este tipo de estudios debería otras variables conocidas por la literatura como intermedias, y que son métricas que permiten entender el proceso por el cual se generan las ganancias. Dentro de dichas métricas se pueden encontrar indicadores como el awareness de marca, producto o categoría, la lealtad del consumidor, diferentes medidas de engagement online, entre otras mediciones. 


\section{Referencias bibliográficas}

Budikova, J. (2014). Discussion: how digital trends are changing the marketing landscape. Central European Business Review. (2), 57-58.

Chaffey, D. y Ellis-Chadwick, F. (2014). Marketing digital. Mexico: Pearson.

Cravens, D. (2006). Strategic Marketing's Global Challenges and Opportunities. Handbook of Business Strategy, (1), 63 -70.

eMarketer (2015). Total Media Ad Spending Growth Slows Worlwide. Recuperado de: http://www.emarketer.com/Article/ Total-Media-Ad-Spending-Growth-SlowsWorldwide/1012981

EMIS. (2015). Informes sectoriales en Colombia. Recuperado de http://www. securities.com/emis/

Frösen, J., Henrikki, T., Jaakkola, M. y Vassinen, A. (2013). Marketing performance assesment systems and the business context. European journal of marketing, (5/6), 715-737.

García, O. L. (2009). Administración Financiera, fundamentos y aplicaciones. Cuarta Edición. Bogotá: Editorial Oscar León García, 4ed.

IAB Colombia (2015). Reporte Sobre La Inversión en Medios Digitales en Colombia. Recuperado de http://www.iabcolombia. com/guias-y-estandares/informe-anual/
IAB Colombia (2014). Reporte sobre la inversión en medios digitales en Colombia. recuperado de http://www.iabcolombia. com/guias-y-estandares/informe-anual/

IAB Colombia (2013). Reporte sobre la inversión en medios digitales en Colombia. Recuperado de http://www.iabcolombia. com/guias-y-estandares/informe-anual/

Islamoglu, M., \& Celik, N. (2015). Financial performance determinants of paper and paper products firms listed in Borsa Istanbul. International Journal of Economics and Finance, 7(4), 233-243.

Kotler, P. y Keller, K (2012). Dirección de Marketing. México: Prentice Hall.

Pimenta da Gamma, A. (2011). An expanded model of marketing Performance, Marketing Intelligence and Planning, (7), 643-661.

Stone, D. y Woodcock, N. (2014). Interactive, direct and digital marketing. Journal of Research in Interactive Marketing, (1), 4- 17.

Strauss, J. y Frost, R. (2012). E-Marketing. New Jersey: Pearson.

Uribe, F., Rialp, J. y Llonch, J. (2013). El uso de las redes sociales digitales como Herramienta de Marketing en el Desempeño Empresarial. Cuadernos de Administración, 26 (47), 205-231 
